DOCUMENTS

pour l'histoire

des techniques
Documents pour l'histoire des techniques

Nouvelle série

$17 \mid 1^{\text {er }}$ semestre 2009

L'invention technique et les figures de l'inventeur (XVIII $-X^{\mathrm{e}}$ siècles)

\title{
Luisa Dolza, Storia della tecnologia
}

Bologna, Universale Paperbacks Il Mulino, 2008, 241 pages.

\section{Romano Nanni}

Traducteur : Frédérique Malbos

\section{(2) OpenEdition}

\section{Journals}

Édition électronique

URL : http://journals.openedition.org/dht/583

DOI : $10.4000 /$ dht.583

ISSN : 1775-4194

Éditeur :

Centre d'histoire des techniques et de l'environnement du Cnam (CDHTE-Cnam), Société des élèves du CDHTE-Cnam

Édition imprimée

Date de publication : 31 mars 2009

Pagination : 231-233

ISBN : 978-2-95-30779-3-3

ISSN : 0417-8726

\section{Référence électronique}

Romano Nanni, « Luisa Dolza, Storia della tecnologia », Documents pour I'histoire des techniques [En ligne], $17 \mid 7^{\text {er }}$ semestre 2009, mis en ligne le 27 septembre 2010, consulté le 24 septembre 2020. URL : http://journals.openedition.org/dht/583 ; DOI : https://doi.org/10.4000/dht.583

Ce document a été généré automatiquement le 24 septembre 2020.

(c) Tous droits réservés 


\title{
Luisa Dolza, Storia della tecnologia
}

Bologna, Universale Paperbacks Il Mulino, 2008, 241 pages.

\author{
Romano Nanni
}

Traduction : Frédérique Malbos

\section{RÉFÉRENCE}

Luisa Dolza, Storia della tecnologia, Bologna, Universale Paperbacks Il Mulino, 2008, 241

pages.

1 La collection Universale Paperbacks s'adresse à un vaste public auquel elle veut offrir, en livres de poche, des synthèses actualisées sur un sujet ou une matière. Quand le sujet est démesuré comme celui d'une histoire de la technique de l'Antiquité à nos jours, il semble difficile d'éviter des choix draconiens dans l'exposition. Bruno Jacomy par exemple, dans un travail présentant le même genre de contraintes, Une histoire des techniques (Seuil, 1990), avait choisi d'offrir pour chaque époque un bref aperçu, un objet technique et une figure de "technicien " considérés comme emblématiques de l'époque. Luisa Dolza a choisi un fil conducteur principal, celui de l'histoire de la modification du statut et de l'image sociale de l'inventeur, ou de l'ingegno ${ }^{1}$, et notamment la formation du concept de propriété intellectuelle. En choisissant ce point de vue, Luisa Dolza fait œuvre de synthèse et de divulgation des orientations et des résultats de la recherche sur diverses périodes marquées par l'intérêt pour les machines et l'invention technique - une historiographie qualifiée souvent d'internaliste - tout en liant ce thème à celui des conditions du développement économique.

2 L'Antiquité gréco-romaine et le Moyen Âge chrétien semblent fondamentalement attachés à l'idée d'invention comme don divin, malgré quelques failles et différentiations - que l'auteure signale. A l'époque de Virgile entre autres, on était convaincu du caractère exceptionnel des œuvres d'ingénierie par rapport à la caducité universelle de toutes choses : seules les œuvres du génie pouvaient laisser des traces plus durables, monuments de la mémoire pour la postérité. Conviction résumée dans 
l'expression Vivitur ingenio !,qui deviendra emblématique d'une image de l'inventeur à la Renaissance puis au XIX ${ }^{e}$ siècle. La civilisation technique antique et celle du Moyen Âge restent malgré tout marquées par l'anonymat du monde des techniciens : machines et instruments, quand ils n'étaient pas un don divin, n'étaient pas considérés comme des inventions à l'état pur mais le fruit d'une attitude auto-élaboratrice de la tradition technique, fondée sur l'imitation de la nature; vision qui se transformera au Moyen Âge dans le concept de mécanique pratique comme science adultérine, impure, dans le contexte d'une codification de la hiérarchie des arts qui attribue aux art mécaniques un rang inférieur.

3 Il faut dire que la vivacité de la technique médiévale - sur laquelle l'auteure s'arrête tout particulièrement - débouchera sur certaines évolutions idéologiques, de Ugo di San Vittore à Roger Bacon. Mais on ne pouvait trouver à cette époque le concept de propriété intellectuelle de l'œuvre, qui commencera à se former du XV ${ }^{e}$ au XVII ${ }^{e}$ siècle - les chapitres centraux du livre y sont consacrés - et où la narration se concentre de façon plus incisive sur certaines conditions institutionnelles et sociales du progrès technique, évoquées dans une perspective historiographique, admise couramment, celle de la longue durée ; il s'agit des conditions autoritaires de gestion de l'innovation technique, des modalités de transmission, de la protection et de la circulation des savoir-faire, des retombées sur la structure du système technique de certaines filières comme celle de l'artillerie et des techniques de fusion, et enfin de la rhétorique publique de l'ingegno.

4 A propos de la circulation des savoir-faire, l'auteure passe en revue l'éventail des problèmes liés au contrôle et à la mobilité de la main d'œuvre qualifiée, tout comme le rôle de l'imprimé et notamment des théâtres de machines. Le clou de la narration est la question du rapport entre la naissance de la pratique du "privilège », ou «lettre patente ", les comportements des corporations, les stratégies des Etats et des pouvoirs civils à ce sujet. A partir de la loi vénitienne sur les privilèges industriels de 1474, l'octroi de lettres patentes ou autorisations d'exploitation économique d'une invention deviennent monnaie courante en Europe - notamment en France, en Italie et en Angleterre, bien que selon des modes très variés. Cette pratique comprend aussi l'octroi de privilèges pour la publication de livres de machines et d'inventions, y compris la reconnaissance de paternité aux auteurs d'œuvres présentées comme une redécouverte et un recueil d'exemples et de savoirs anciens, héritiers d'une pratique inaugurée à l'époque de l'humanisme. Et c'est dans le cadre des privilèges que commence à se former - bien que par vagues successives et non sans heurts - le concept de propriété de l'œuvre et de droit sur celle-ci. Parallèlement à cette histoire, l'auteure brosse avec brio le tableau des discussions, des images et des aspects de rhétorique publique sur la technique, qui divergent parfois, issus de beaucoup d'auteurs qui de plus en plus se mesureront au thème aux $\mathrm{XVI}^{\mathrm{e}}$ et $\mathrm{XVII}^{\mathrm{e}}$ siècles, de Besson à Ceredi, Vives, Agricola, Ramelli, Strada, Del Monte, Bacon, Descartes, Hobbes et Galilée.

On peut se demander si cette approche ne laisse pas un peu dans l'ombre des aspects essentiels de la réalité des savoirs et des techniques effectives. Par exemple, l'espace consacré au rôle des théâtres de machines et à l'imprimé peut être compréhensible dans une optique de longue durée, et dans un esprit de la Mitteleuropa. Mais les manuscrits et les dessins techniques ont eu la vie plus longue et un rôle plus important et fonctionnel que ce qu'il paraît de cet angle de vue, et peut-être que la pratique effective des «mécaniciens »- comme celle des architectes, dont les compétences ont 
englobé longuement celles des ingénieurs - passa à travers ce type d'écrit plutôt que par les livres imprimés. Il est probable que le parti pris de l'optique historiographique du privilège fait pencher vers une histoire de ce qui est pour ainsi dire « visible » sous le profil officiel de l'invention technique, bien que l'auteure signale plusieurs fois en connaissance de cause que les privilèges n'épuisèrent pas du tout les voies du développement technique.

6 En effet, une fois révolue l'heure de gloire des théâtres de machines, on signale l'essor d'une nouvelle dialectique. On pourrait dire dans un certain sens qu'au XVIII siècle il est advenu pour le système des privilèges ce qui est arrivé de nos jours avec les bulles spéculatives qui ont prospéré autour du marché de la new economy. Dès la fin du XVII siècle, les privilèges engendrent des investissements croissants, y compris spéculatifs, dans l'espoir de dividendes, investissements qui à un certain moment partent en vrille face à l'exiguïté des résultats, entraînant une réaction de méfiance et de discrédit vis-àvis de l'inventeur également. L'invention ne semble plus aussi héroïque et positive qu'à la grande époque des théâtres de machines. C'est dans ce contexte que l'auteure retrace les développements du XVIII siècle français le long de deux voies parallèles, celle de la politique étatique et celle de l'idéologie. D'un côté l'état français commence à reconnaître l'invention comme faisant partie du patrimoine national ; l'administration publique, surtout à partir de 1762, s'oriente, pour l'octroi des privilèges sur les inventions, vers des critères d'utilité économique plutôt que de nouveauté, et vers un droit de regard pour le concessionnaire quant à la diffusion et à la transmission de son propre savoir-faire. L'inventeur est ainsi soumis au jugement et au contrôle de hiérarchies bureaucrates, scientifiques et corporatives qui relèveront par la suite de l'Académie royale des sciences. D'autre part, les positions de Diderot et de l'Encyclopédie sont fondées sur la conviction que l'œuvre de l'inventeur, contrairement à celle de l'artiste, n'est pas une création originale absolue dont l'auteur puisse revendiquer la paternité et les fruits exclusifs, mais plutôt une redécouverte et une adaptation de ce que la nature fait déjà toute seule, et est de plus le fruit des innombrables apports du travail collectif des artisans. Il y a donc un refus de la reconnaissance de la propriété exclusive de l'invention à l'inventeur, et des honneurs qui s'y rattachent. L'objectif de l'idéologie des encyclopédistes est par contre de divulguer au maximum les découvertes, pour en augmenter l'utilité publique et favoriser le progrès technique. Ceci dit, la situation française finit également par produire un mouvement conflictuel en sens inverse. La colonisation du territoire de l'invention de la part de l'administration publique finit par imposer la figure publique de l'inventeur qui de son côté nourrit une impatience croissante vis-à-vis du contrôle des académiciens, tout comme les artisans du Piémont regardent avec mépris les «inventions de salon» des scientifiques, comme les automates et choses de la sorte. Sur cela vient se greffer une tension qui finit par déboucher sur la loi de 1791 par laquelle il est décrété que toute idée nouvelle, même utile à la société, appartient avant tout à celui qui l'a conçue.

7 Ce qui précède fut un versant très français de l'histoire. De l'autre côté de la Manche, s'était développé un rapport avec l'innovation technique moins dépendant du système des lettres patentes; et surtout à partir de la Révolution industrielle, cela conduira à un rapport plus direct avec l'entreprise et par là avec la vérification in re de l'utilité de l'invention. Le cas de la machine à vapeur de James Watt est emblématique à cet égard. $\mathrm{Au}$ privilège enregistré en 1769 puis prolongé jusqu'à 1800 s'ajoutait une véritable stratégie d'exploitation économique de l'invention fondée sur plusieurs piliers: la création, avec Matthew Boulton d'une entreprise de conception et de contrôle de la 
construction des machines, qui tirait ses profits des royalties sur le fonctionnement de ces dernières; l'extension assez large de la couverture assurée par le privilège, qui rendait difficiles les innovations de la part d'autres personnes sur la même machine et assurait aussi le contrôle de toute une filière technique ; les efforts de Watt lui-même pour perfectionner et développer ses propres inventions; une politique d'image visant sciemment à valoriser la relation entre le nom de l'inventeur et sa technologie.

D'autre part, cette situation de monopole déclencha aussi toute une série de réactions de la part des entreprises qui à leur tour misèrent sur la publicité et la divulgation des caractéristiques des nouveaux machinismes, en arrivant aussi à renoncer aux privilèges afin de favoriser des processus de perfectionnement continu, comme dans le cas des locomotives à vapeur pour les mines.

C'est au XIX ${ }^{e}$ siècle que les inventeurs seront consacrés sans réserves comme des héros, d'abord avec les Romantiques puis avec la culture positiviste. L'époque est celle qui voit naître les chemins de fer et les grands ponts, la vapeur et l'électricité, la révolution industrielle, les grandes expositions de la science et de la technique ainsi que les grands musées scientifiques, le développement moderne des écoles polytechniques et d'ingénierie et des hagiographies de l'inventeur. Dans la deuxième moitié du siècle, on assiste aussi à la réouverture d'un débat sur l'utilité ou non du système des brevets, qui aboutira plus tard à la distinction schumpétérienne entre invention et innovation. Jusqu'à la fin du XIX siècle, on aura la perception, non sans voix discordantes, d'un lien incontournable et vertueux entre technique et progrès, qui se décomposera progressivement au cours $\mathrm{du} \mathrm{XX}^{\mathrm{e}}$ siècle, face aux dérives inquiétantes du développement technologique semblant presque pousser à récupérer l'ambivalence de l'image de la technique qui fut celle de l'Antiquité. Mais ceci est une autre histoire.

\section{NOTES}

1. Ingegno en italien est à la fois le génie, l'ingéniosité et l'ingénierie.

\section{AUTEURS}

\section{ROMANO NANNI}

Bibliothèque et Musée Léonard de Vinci, Vinci 